\title{
Increased expression of the NLRP3 inflammasome components in patients with Behçet's disease
}

\author{
En Hyung Kim ${ }^{1,3}$, Mi-Jin Park', Sun Park ${ }^{2}$ and Eun-So Lee ${ }^{1 *}$
}

\begin{abstract}
Background: Behçet's disease (BD) is a systemic inflammatory disease with manifestations including recurrent oral and genital ulcerations, and vasculitis involving the skin, mucosa, joints, eyes, veins, arteries, nervous and gastrointestinal systems. BD is seen as a disease at the crossroad between autoimmune and autoinflammatory syndromes, possibly triggered by an aberrant response to infectious stimuli. The relevance of Gram negative bacteria-mediated oral inflammation with the increased expression of NACHT, LRR, and PYD domains-containing protein 3 (NLRP3), leading to systemic inflammation, prompted us to investigate the expression of NLRP3 inflammasome components and its link with IL-1 $\beta$ hypersecretion.

Findings: When peripheral blood mononuclear cells (PBMCs) from 15 active, 15 stable BD patients and 15 healthy volunteers were stimulated, the basal and LPS-induced expressions of NLRP3 inflammasome components were significantly increased at both mRNA and protein levels in BD patients compared to healthy controls. Also, increased expression of NLRP3 and ASC was observed in 25 BD skin lesions compared to 25 erythema nodosum patients. Compatible with this, secretion of IL-1 $\beta$ by PBMCs stimulated with LPS alone or LPS plus ATP was increased in BD compared to healthy controls, which was suppressed by caspase-1 inhibitor.
\end{abstract}

Conclusion: Our findings suggest the possible link between increased IL-1 $\beta$ secretion and increased expression of NLRP3 inflammasome components in BD patients with skin manifestations.

Keywords: Behçet's disease, Inflammasome, NLRP3, Interleukin-1 $\beta$

\section{Introduction}

Behçet's disease (BD) is a systemic inflammatory disease with manifestations including recurrent oral and genital ulcerations, and vasculitis involving the skin, mucosa, joints, eyes, veins, arteries, nervous and gastrointestinal systems. BD is currently seen as a disease at the crossroad between autoimmune and autoinflammatory syndromes, possibly triggered by an aberrant response to infectious stimuli [1]. Oral microbial flora have long been implicated in the pathogenesis of BD [2]. However, it is not clear how infection induces an immune response and initiates the development of BD. Recently it was shown that NACHT, LRR, and PYD domains-containing protein 3 (NLRP3) inflammasome is upregulated when infected by

\footnotetext{
* Correspondence: esl@ajou.ac.kr

${ }^{1}$ Department of Dermatology, Ajou University School of Medicine, 164

Worldcup-ro, Yeongtong-Gu, Suwon 443-380, South Korea

Full list of author information is available at the end of the article
}

Porphyromonas gingivalis, a G(-) bacteria, and in some cases lead to induction and sustained aortic or gingival inflammation [3]. Also, peptidoglycan and LPS induced IL$1 \beta$ via the TLR2/4 and reactive oxygen species-NLRP3 inflammasome-dependent pathways in ocular BD [4]. As oral ulcer precede BD for many years and EN like lesions, one of most common skin manifestions in BD, which are characterized by septal and lobular panniculitis and most importantly vasculitis, we investigated whether NLRP3 inflammasome expression is increased at the mRNA and protein levels in BD patients with skin manifestations. Also, we studied whether IL- $1 \beta$ production was mediated through NLRP3 inflammasome dependent pathway in BD.

\section{Methods \\ Patients}

Blood samples were taken from 15 active, 15 stable BD patients and 15 healthy volunteers (HC) (Table 1). All

\section{Biomed Central}

(C) 2015 Kim et al. This is an Open Access article distributed under the terms of the Creative Commons Attribution License (http://creativecommons.org/licenses/by/4.0), which permits unrestricted use, distribution, and reproduction in any medium, provided the original work is properly credited. The Creative Commons Public Domain Dedication waiver (http:// creativecommons.org/publicdomain/zero/1.0/) applies to the data made available in this article, unless otherwise stated. 
Table 1 Characteristics of Behçet's disease (BD) patients and healthy controls

\begin{tabular}{|c|c|c|c|}
\hline Demographics & Active BD & Inactive $\mathrm{BD}$ & $\begin{array}{l}\text { Healthy } \\
\text { control }\end{array}$ \\
\hline Age $($ mean $\pm S D)$ & $41.86 \pm 7.54$ & $43.6 \pm 8.04$ & $38.93 \pm 6.94$ \\
\hline Sex (male:female) & $6: 9$ & $7: 8$ & $7: 8$ \\
\hline $\begin{array}{l}\text { Clinical characteristics } \\
\text { of BD patients }\end{array}$ & $\begin{array}{l}\text { Case } \\
\text { number (\%) }\end{array}$ & & \\
\hline \multicolumn{4}{|l|}{ Major symptoms } \\
\hline Oral ulcer & $15(100)$ & & \\
\hline Genital ulcer & $10(67)$ & & \\
\hline Skin lesion & $10(67)$ & & \\
\hline Ocular lesion & $4(27)$ & & \\
\hline \multicolumn{4}{|l|}{ Minor symptoms } \\
\hline Arthritis & $2(13)$ & & \\
\hline Gl involvement & $1(7)$ & & \\
\hline Epididymitis & 0 & & \\
\hline Cardiovascular & $1(7)$ & & \\
\hline CNS involvement & $1(7)$ & & \\
\hline \multicolumn{4}{|l|}{ Medication } \\
\hline Immunosuppressive agent ${ }^{a}$ & $6(40)$ & $0(0)$ & \\
\hline Anti-inflammatory drugs ${ }^{b}$ & $9(60)$ & $7(46)$ & \\
\hline
\end{tabular}

${ }^{a}$ Methylprednisolone 8-24 mg/d

${ }^{\mathrm{b} C}$ Colchicine $1.2 \mathrm{mg} / \mathrm{d} \pm$ minocycline $100 \mathrm{mg} / \mathrm{d} \pm$ pentoxiphylline $800 \mathrm{mg} / \mathrm{d} \pm$ sulfasalazine $1000 \mathrm{mg} / \mathrm{d}$

patients consisted of BD patients who presented themselves for the first time or were monitored at the Department of Dermatology, Ajou University Hospital. BD patients met the Diagnostic criteria of the BD Research Committee of Japan. The active group patients had at least one of the BD symptoms despite the treatment and inactive group patients were in well-controlled states. Informed consent was obtained prior to the study. This study was approved by the Institutional Review Board (IRB no.: AJIRB-GN3-07-098, AJIRB-GGEN-GEN-10-119).

\section{Immunohistochemistry}

Six $\mathrm{mm}$ punch skin biopsies of erythema nodosum (EN)-like lesions of $25 \mathrm{BD}$ and $25 \mathrm{EN}$ patients were performed. Formalin-fixed and paraffin-embedded tissues of EN-like lesions in BD were cut $(3-\mu \mathrm{m}$ thickness) and mounted onto slides. Specimens were deparaffinated and endogenous peroxidase activity was blocked by $3 \%$ $\mathrm{H}_{2} \mathrm{O}_{2}$ in methanol for $15 \mathrm{~min}$ at room temperature. After rinsing in phosphate-buffered saline (PBS) for $10 \mathrm{~min}$, the nonspecific binding sites were blocked by blocking solution for $10 \mathrm{~min}$ at room temperature and all specimens were incubated with polyclonal antibodies against NLRP3 (1:50 dilution, mouse, Alexis Biochemicals, San diego, CA, USA) and ASC (1:100 dilution, rabbit, Lifespan bioscience, Seattle, WA) for $30 \mathrm{~min}$ at room temperature. Next, HRP polymer (Thermo scientific, Fremont, CA, USA) was applied and incubated for $30 \mathrm{~min}$ at room temperature. After washing in PBS for $10 \mathrm{~min}$, bound antibodies were visualized by incubation with AEC chromogen system (Thermo scientific, Fremont, CA, USA). Slides were counterstained with hematoxylin. Negative controls were isotype matched. The image was analyzed using Image Pro Plus Version 4.5 (Media Cybertics Co., MD, U.S.A.) (Additional file 1).

\section{Quantitative real time PCR}

Peripheral blood mononuclear cells (PBMCs) were prepared from heparinized blood samples by Ficoll Hypaque density gradients (Ficoll paque ${ }^{\text {TIM }}$ plus, StemCell Technologies, Vancouver, BC, Canada). PBMCs were stimulated for $4 \mathrm{~h}$ with $100 \mathrm{ng} / \mathrm{ml}$ lipopolysaccharide (LPS; Sigma-Aldrich). After $4 \mathrm{~h}$, RPMI containing $1 \mathrm{mM}$ adenosine 5-triphosphate (ATP; Sigma-Aldrich) was added to the cells for another $15 \mathrm{~min}$ (LPS/ATP). In separate experiments, $20 \mu \mathrm{M}$ zYVAD(Ome)-FMK an irreversible caspase-1 inhibitor (CaspI; Enzo life science, PlymouthMeeting, PA) was added. The method for Quantitative real time PCR is described in Additional file 2.

\section{Western blotting}

Briefly, freshly isolated PBMCs and stimulated PBMCs were harvested and lysed in RIPA buffer (Sigma-Aldrich, St. Louis, MO, USA) containing protease inhibitors. Cell extracts were run on Bolt ${ }^{\text {tm }} 4-12 \%$ Bis-Tris Plus Gel (life technologies, Carlsbad, CA, USA) and transferred onto polyvinylidene difluoride membranes (Merck Milipore, Darmstadt, Germany). Following transfer, the membrane was blocked overnight at room temperature with PBS/ $0.2 \%$ Tween-20/5\% skim milk. Blots were incubated with primary antibodies anti-NLRP3, anti-apoptosis-associated speck-like protein containing a CARD (ASC), and anticaspase-1 antibodies (Abcam, Cambridge, MA). The membranes were incubated in a solution containing an appropriate secondary $\mathrm{Ab}$ (either anti-rabbit IgG or antimouse IgG $\mathrm{Ab}$ ) linked to horseradish peroxidase (Invitrogen). Bands were visualized with Immobilon Western Chemiluminescent HRP Substrate (Merck Milipore, Darmstadt, Germany) (Additional file 2).

\section{ELISA for IL-1 $\beta$}

Total IL- $1 \beta$ and mature IL- $1 \beta$ level in the supernatants was measured with a commercial ELISA kit from R\&D Systems according to the manufacturer's protocols.

\section{Statistics}

The data are presented as mean \pm standard deviation (S.D.). Data were analyzed by one-way analysis of variance followed by the Scheffé test for overall multiple comparisons among $\mathrm{HC}$, stable BD, active BD. Student's 
$t$-test was used for comparison between two groups. SPSS 17.0 (SPSS Inc., Chicago, IL) was used. A $p$-value $<0.05$ was considered to indicate statistical significance.

\section{Results and discussion}

Previous reports show NLRP3 inflammasome expression is increased in inflammatory diseases [5]. We investigated the protein and mRNA levels of the different components of NLRP3 inflammasomes, and NLRP1 for comparison. The protein expression of NLRP3 inflammasome components in PBMCs of BD patients was analyzed by western blotting (Fig. 1a and b). The mean values of normalized NLRP3, ASC and caspase-1 protein levels were significantly up-regulated in active and stable $\mathrm{BD}$ compared with HC. NLRP3, ASC and caspase-1 mRNA expression was significantly up-regulated in active and stable BD compared with HC (Fig. 1c). Next, we examined skin lesions for in situ expression of NLRP3 and ASC to correlate our in vitro findings. NLRP3 and ASC in skin lesions of BD and control EN patients were detected by immunohistochemistry. NLRP3 and ASC was mostly expressed by CD68+ macrophages/monocytes (Additional file 3). Image analysis showed that NLRP3 and ASC expression was significantly increased in BD skin lesions (Fig. 1d). These findings show that mRNA and protein levels of NLRP3 inflammasome components are increased in BD patients.

Toll-like receptor signaling induces transcription of NLRP3 and IL-1 $\beta[6,7]$. NLRP3 inflammasome is activated by canonical stimuli like ATP or Nigericin and noncanonical stimuli like live gram negative bacteria [8]. Therefore, we checked whether LPS alone, or ATP stimulation after LPS priming (LPS/ATP), affected the expression of NLRP3 inflammasome components in PBMCs of BD patients. The protein levels of, NLRP3, ASC and caspase-1 were higher following LPS stimulation compared to no stimulation in all the groups and

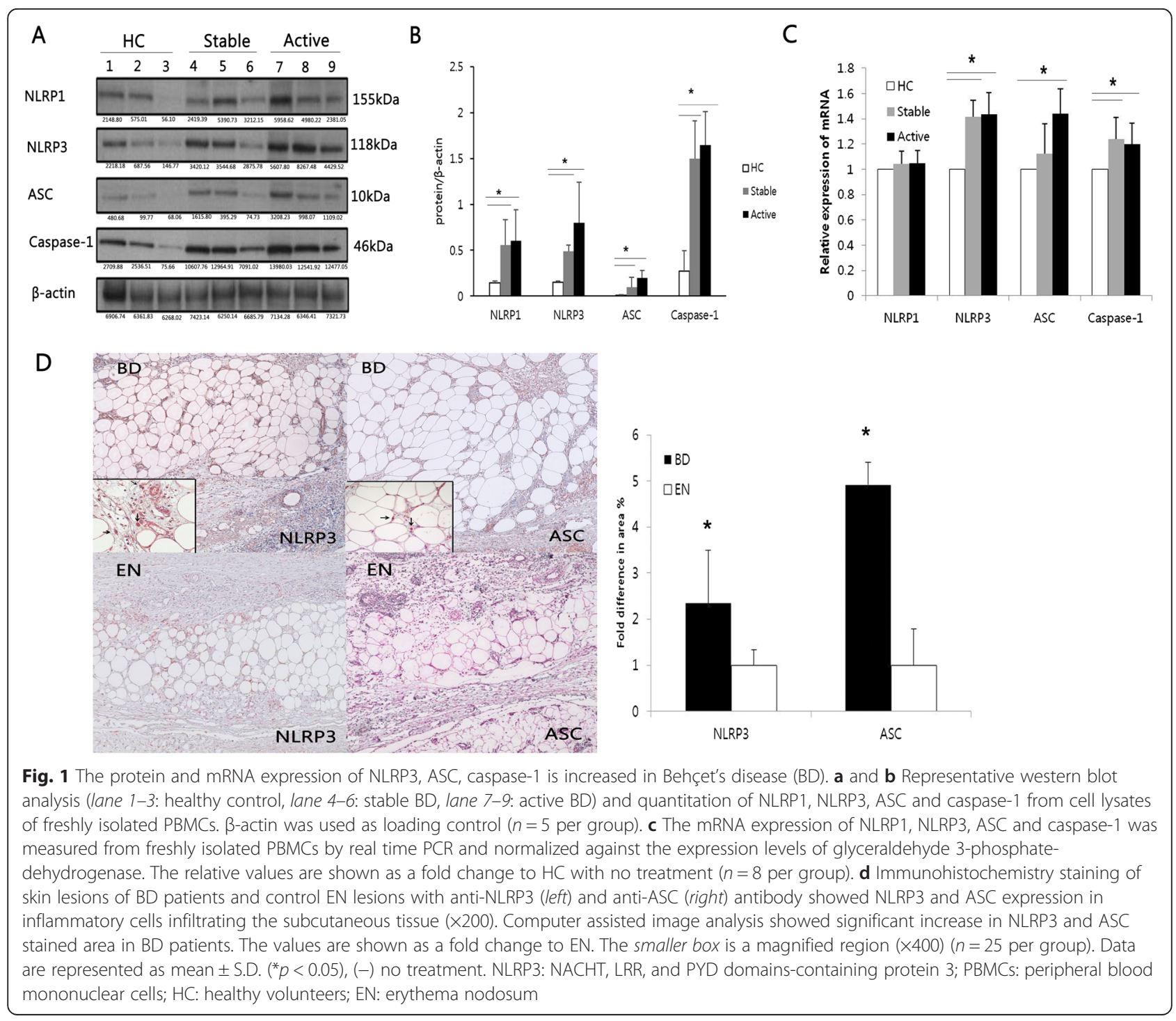


the levels increased significantly in active and stable BD compared to HC (Fig. 2a). Following LPS/ATP stimulation, NLRP3 and ASC protein levels were significantly up-regulated only in active BD compared to HC (Fig. 2a). The mRNA levels of, NLRP3, ASC and caspase-1 were higher after LPS/ATP stimulation compared to single stimulus of LPS in all the groups. Furthermore, they were significantly increased in the presence of LPS or LPS/ATP in active and stable BD compared to $\mathrm{HC}$ (Fig. 2b). These findings show that LPS/ATP stimulation resulted in significantly higher expression of NLRP3 inflammasome component at protein and mRNA levels in PBMCs of BD patients.

To ascertain whether the increased NLRP3 inflammasome components might contribute to increased secretion of IL-1 $\beta$ in BD, we assessed IL- $1 \beta$ secretion by PBMCs stimulated with LPS or LPS/ATP (Fig. 3a and b) In accordance with previous reports [7] showing that peripheral blood monocytes stimulated with LPS release ATP and consequently secrete IL-1 $\beta$, treatment of PBMCs with LPS alone increased IL- $1 \beta$ secretion compared to no stimulation. This effect was suppressed by caspase-1 inhibition and significantly higher in BD compared to HC (Fig. 3a). Additionally, mature IL-1 $\beta$ secretion in the presence of LPS/ATP was significantly higher in active and stable BD than $\mathrm{HC}$ and suppressed by caspase- 1 inhibitor (Fig. 3b). There were significant differences in LPS-induced and LPS/ATP-induced IL-1 $\beta$ mRNA levels between BD and HC (Fig. 3c). However, caspase-1 inhibitor suppressed mature IL-1 $\beta$ secretion in the presence of LPS/ATP without a decrease in mRNA levels (Fig. 3b and c). These findings suggest that stimulation induced IL-1 $\beta$ expression and higher expression of NLRP3 inflammasome components in BD might contribute to increased IL- $1 \beta$ secretion in $\mathrm{BD}$ patients.

The hypothesis of an environmental trigger in BD patients with genetic susceptibilities has long been advocated [2]. These triggers range from infections to molecular mimicry such as heat-shock proteins (HSP), which are synthesized under cellular stress. Inflammasomes are involved in both pathways of pathogen and danger signal sensing, making it a good target for investigation in BD pathogenesis. Presence of a prolonged inflammation such as non-specific (pathergy) or urate induced skin responses suggests that innate and adaptive pathways are more integrated in $\mathrm{BD}$ [9]. The augmented adaptive responses can be the result of persistant oral and skin infections. Given the high frequency of oral ulcer and the decrease of some symptoms with minocycline, oral flora has been implicated in the pathogenesis of BD [10,11]. Recent studies have linked oral infection to inflammasomes leading to sustained inflammation in atherosclerosis [3]. In this study we showed that the mRNA and protein levels of the NLRP3 inflammasome were upregulated in PBMCs of BD patients and this was further supported by in situ findings in skin lesions. This may be a coincidental finding due to tissue damage and release of DAMP, however the increase was more significant compare to RAU, which also shows tissue damage

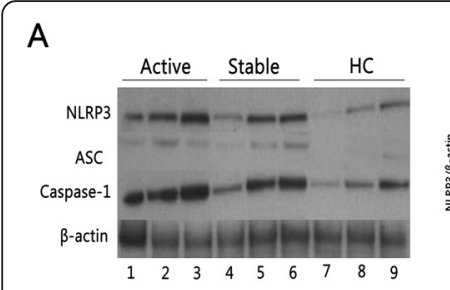

B

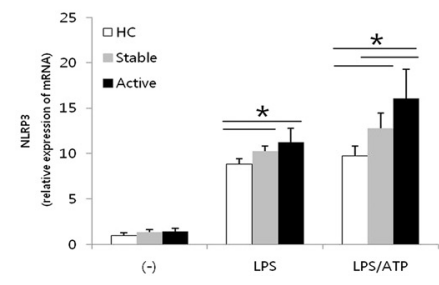

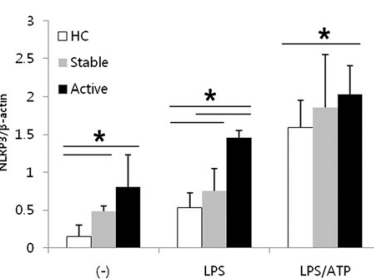
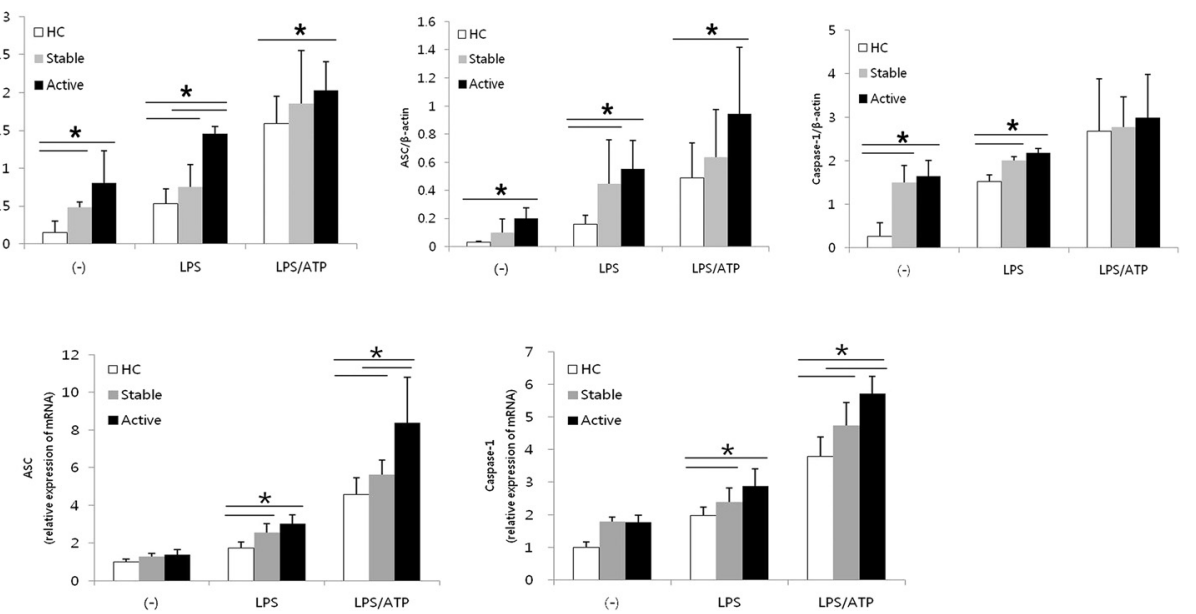

Fig. 2 The induced expression of NLRP3, ASC and caspase-1 is increased in Behçet's disease (BD). PBMCs were initially stimulated for 4 h with LPS (100 ng/ml). After $4 \mathrm{~h}$, ATP (1 mM) was added to the cells for another $15 \mathrm{~min}$ (LPS/ATP). a Representative western blot analysis and quantitation of NLRP3, ASC and caspase-1 from cell lysates of stimulated PBMCs. $\beta$-actin was used as loading control. (lane 1,4,7: no treatment, lane 2,5,8: LPS, lane 3,6,9: LPS/ATP) ( $n=5$ per group). b The mRNA expression of NLRP3, ASC and caspase- 1 was measured by real time quantitative RT-PCR and normalized against the expression levels of glyceraldehyde 3-phosphate-dehydrogenase. The relative values are shown as a fold change to $\mathrm{HC}$ with no treatment ( $n=8$ per group). Data are represented as mean \pm S.D. $\left({ }^{*} p<0.05\right),(-)$ no treatment. NLRP3: NACHT, LRR, and PYD domainscontaining protein 3; PBMCs: peripheral blood mononuclear cells; LPS: lipopolysaccharide; ATP: adenosine 5-triphosphate; HC: healthy volunteers 

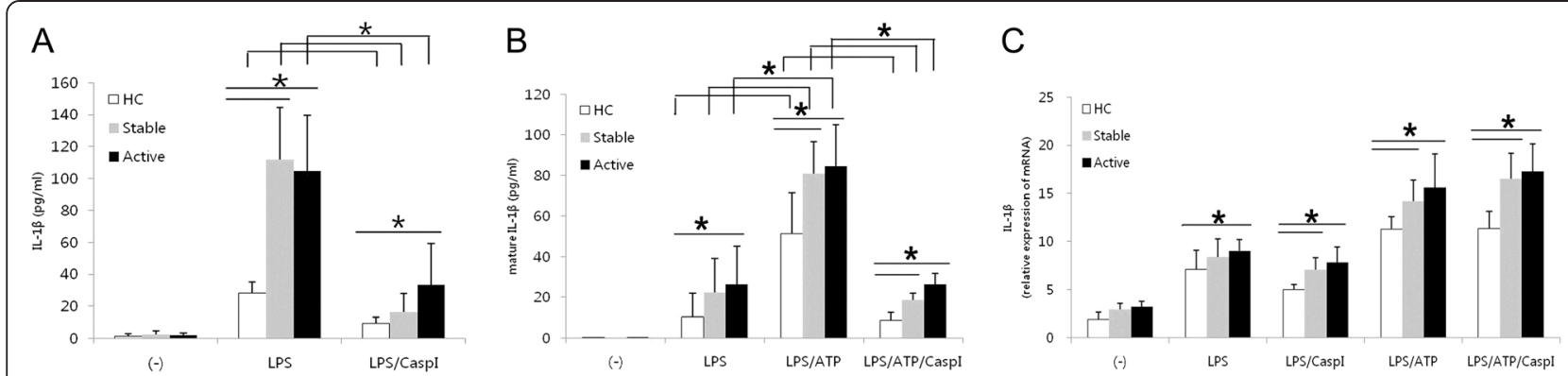

Fig. 3 Caspase-1 inhibits increase of IL-1 $\beta$ secretion by peripheral blood mononuclear cells (PBMCs) following NLRP3 activation. PBMCs were initially stimulated for $4 \mathrm{~h}$ with LPS (100 ng/ml) with or without $20 \mu \mathrm{M}$ zVAD(Ome)-FMK, an irreversible caspase-1 inhibitor (LPS/Caspl). After $4 \mathrm{~h}$, ATP $(1 \mathrm{mM})$ was added to the cells for another $15 \mathrm{~min}$ (LPS/ATP or LPS/ATP/Caspl). a Total IL-1 $\beta$ ( $n=15$ per group) and $\mathbf{b}$ mature processed $\mathrm{IL}-1 \beta$ ( $n=9$ per group) was quantitated in the supernatant of stimulated PBMCs by ELISA. $\mathbf{c}$ The mRNA expression of IL-1 $\beta$ was measured by real time PCR and normalized against the expression levels of glyceraldehyde 3-phosphate-dehydrogenase. The relative values are shown as a fold change to $\mathrm{HC}$ with no treatment $\left(n=8\right.$ per group). Data are represented as mean \pm S.D. $\left({ }^{*} p<0.05\right)$, $(-)$ no treatment. PBMCs: peripheral blood mononuclear cells; NLRP3: NACHT, LRR, and PYD domains-containing protein 3; LPS: lipopolysaccharide; ATP: adenosine 5-triphosphate

(Additional file 4). Thus increase in NLRP3 inflammasomes may have a role in $\mathrm{BD}$ pathogenesis.

Pathways of inflammasome and pathogen-associated molecular pattern such as toll-like receptors (TLRs) intersect as both are sensors of bacterial products. We have previously shown that TLR2 expression increases at both the mRNA and protein levels, along with TLR4 expression at the mRNA level in active BD patients [12]. LPS from microorganisms could initiate IL- $1 \beta$ synthesis process through TLRs and the inflammasomes. The extracellular ATP released from stressed or infected cells in oral tissue may further activate NLRP3 inflammasomes leading to IL-1 $\beta$ maturation and release. The released IL$1 \beta$ can activate the release of other proinflammatory cytokines. In this study we showed that the components of the NLRP3 inflammasome pathway along with IL-1 $\beta$ mRNA level were higher after LPS/ATP challenge compared to LPS stimulation only in PBMCs of BD patients. The upregulation of components of the NLRP3 inflammasome pathway along with IL-1 $\beta$ mRNA corresponded with increased secretion of IL-1 $\beta$. Also, IL-1 $\beta$ decreased when caspase-1 inhibitor was added.

IL-1 $\beta$ synthesis, maturation, and secretion are tightly regulated by Toll-like receptor (TLR) signaling and inflammasome activation [13]. IL-1 $\beta$ has significant role in BD pathomechanism. Specific IL-1 $\beta$ gene polymorphisms cause increased susceptibility to BD and IL- $1 \beta$ blockade with recombinant anti-IL-1 $\beta$ antibody gevokizumab has been used for BD treatment [14]. Thalidomide, an anti-inflammatory drug, used to treat $\mathrm{BD}$, was found to decrease IL- $1 \beta$ through caspase-1 inhibition [15]. Therefore understanding the mechanism of NLRP3 in BD will not only help to elucidate their role in host responses to infectious agents and to danger signals, but may also contribute to the development of novel treatment of BD.

\section{Conclusion}

In this study, we showed that expression of NLRP3 inflammasome components were increased in BD PBMCs and skin lesions. Also, IL-1 $\beta$ secretion was increased in BD PBMCs when NLRP3 inflammasome components were elevated and suppressed when caspase-1 inhibitor was added. Our findings suggest the possible link between increased IL-1 $\beta$ secretion and increased expression of NLRP3 inflammasome components in BD patients with skin manifestations.

\section{Additional files}

Additional file 1: Image analysis of immunohistochemical staining. Image signals were recorded on a personal computer and evaluated using Image Pro Plus Version 4.5 (Media Cybernetics Co., Silver Spring, MD, USA). For each staining, we established a standard for antibody (Ab) positivity and applied the same standards to the samples. The stained area per total area was measured and the ratio of positive Ab area (yellow) to the septal and lobular panniculitis area (green) was calculated. Each measurement was evaluated under constant magnification $(\times 200)$. As inflammation presents mainly in and around the septa, fat lobules were left out when calculating the area. The image analysis was performed on a representative area of each specimen and repeated three times by three examiners and the mean was used for evaluation. Finally the data was expressed as fold difference between EN-like lesion and EN.

Additional file 2: Detailed method for Quantitative real time PCR and Western blotting.

Additional file 3: Erythema nodosum-like lesions are infiltrated with CD3+ T lymphocytes, CD68+ monocytes and macrophages, and Myeloperoxidase (MPO) + neutrophils. Immunohistologic staining was performed on formalin-fixed and paraffin-embedded tissues of EN-like lesions with a panel of antibodies directed against mononuclear cell antigens using an indirect avidin-biotin immunoperoxidase technique. The degree of inflammatory cell infiltration, which also showed positivity to NLRP3 in serial section, were graded on a semi-quantitative scale of $0-4$ : 0 , absent; 1 , minimal; 2, mild; 3, moderate; 4 , marked staining. All slides were evaluated by three examiners in a blinded fashion and the average score was calculated for each section. (A) anti-CD3 (1:200 dilution, mouse, Novocastra, Newcastle, UK), (B) anti-CD20 (1:300 dilution, mouse, Dako, Denmark), (C) anti-CD68 (1:80 dilution, mouse, Novocastra, 
Newcastle, UK), (D) anti-MPO (1:600 dilution, rabbit, NeoMarker, Fremount, CA, USA) antibody $(\times 200)(n=25)$. The semi-quantitative analysis of inflammatory cell infiltration was as follows; CD3+ T lymphocytes: $2.01 \pm$ 1.11, CD20+ B lymphocytes: $0.75 \pm 1.05$, CD68+ monocytes/macrophages: $3.14 \pm 0.65, \mathrm{MPO}+$ neutrophils: $2.69 \pm 1.27$. Data are represented as mean \pm S.D.

Additional file 4: The induced expression of NLRP3, ASC, caspase-1 and IL-1 $\beta$ is increased in Behçet's disease (BD) compared to healthy control or recurrent apthous ulcer patients (RAU). PBMCs were initially stimulated for $4 \mathrm{~h}$ with LPS $(100 \mathrm{ng} / \mathrm{ml})$ with or without $20 \mathrm{\mu M}$ zYVAD(Ome)-FMK, an irreversible caspase-1 inhibitor (LPS/Caspl). After $4 \mathrm{~h}$, ATP (1 mM) was added to the cells for another $15 \mathrm{~min}$ (LPS/ATP or LPS/ATP/Caspl). (A-D) The mRNA expression of NLRP3, ASC, caspase-1 and IL-1 $\beta$ was measured by real time quantitative RT-PCR and normalized against the expression levels of glyceraldehyde 3-phosphate-

dehydrogenase. The relative values are shown as a fold change to $\mathrm{HC}$ with no treatment ( $n=8$ per group). (E) Total IL-1 $\beta$ ( $n=15$ per group) was quantitated in the supernatant of stimulated PBMCs by ELISA. Data are represented as mean \pm S.D. $\left({ }^{*} p<0.05\right),(-)$ : no treatment; LPS: lipopolysaccharide; ATP: adenosine 5-triphosphate; Caspl: caspase-1 inhibitor; HC: healthy volunteers, RAU: Recurrent apthous ulcer (disease control).

\section{Abbreviations}

ATP: Adenosine 5-triphosphate; BD: Behçet's disease; EN: Erythema nodosum; HC: Healthy volunteers; LPS: Lipopolysaccharide; RAU: Recurrent apthous ulcer; NLRP3: NACHT, LRR, and PYD domains-containing protein 3; PBMCs: Peripheral blood mononuclear cells; TLRs: Toll-like receptors.

\section{Competing interests}

The authors declare that they have no competing interests. inflammasome-dependent pathways is involved in ocular Behcet's disease. Invest Ophthalmol Vis Sci. 2013;54:402-14.

5. Mitroulis I, Skendros P, Ritis K. Targeting IL-1 beta in disease; the expanding role of NLRP3 inflammasome. Eur J Intern Med. 2010;21:157-63.

6. Qiao Y, Wang P, Qi J, Zhang L, Gao C. TLR-induced NF-kappaB activation regulates NLRP3 expression in murine macrophages. FEBS Lett. 2012;586:1022-6.

7. Netea MG, Simon A, van de Veerdonk F, Kullberg BJ, Van der Meer JW, Joosten LA. IL-1beta processing in host defense: beyond the inflammasomes. PLoS Pathog. 2010;6, e1 000661.

8. Bauernfeind F, Hornung V. Of inflammasomes and pathogens-sensing of microbes by the inflammasome. EMBO Mol Med. 2013;5:814-26.

9. Gogus F, Fresko I, Elbir Y, Eksioglu-Demiralp E, Direskeneli H. Oxidative burst response to monosodium urate crystals in patients with Behcet's syndrome. Clin Exp Rheumatol. 2005;23 Suppl 38:S81-5.

10. Kaneko F, Oyama N, Nishibu A. Streptococcal infection in the pathogenesis of Behcet's disease and clinical effects of minocycline on the disease symptoms. Yonsei Med J. 1997;38:444-54.

11. Calguneri M, Kiraz S, Ertenli I, Benekli M, Karaarslan Y, Celik I. The effect of prophylactic penicillin treatment on the course of arthritis episodes in patients with Behcet's disease. A randomized clinical trial. Arthritis Rheum. 1996:39:2062-5.

12. Do JE, Kwon SY, Park S, Lee ES. Effects of vitamin D on expression of toll-like receptors of monocytes from patients with Behcet's disease. Rheumatology (Oxford). 2008;47:840-8.

13. Dinarello CA. Immunological and inflammatory functions of the interleukin-1 family. Annu Rev Immunol. 2009;27:519-50.

14. Gul A, Tugal-Tutkun I, Dinarello CA, Reznikov L, Esen BA, Mirza A, et al. Interleukin-1 beta-regulating antibody XOMA 052 (gevokizumab) in the treatment of acute exacerbations of resistant uveitis of Behcet's disease: an open-label pilot study. Ann Rheum Dis. 2012;71:563-6.

15. Keller M, Sollberger G, Beer HD. Thalidomide inhibits activation of caspase-1. J Immunol. 2009;183:5593-9.

\section{Authors' contributions}

EH Kim participated in the design and coordination of the study, carried out the immunohistochemistry and RT-PCR, performed the statistical analysis and drafted the manuscript. MJ Park carried out the RT-PCR, western blotting and ELISA. ES LEE and S Sun conceived of the study, and participated in its design and coordination and helped to draft the manuscript. All authors read and approved the final manuscript.

\section{Acknowledgement}

This research was supported by a grant from the Korea Healthcare Technology R\&D Project, Ministry of Health, Welfare and Family Affairs, Republic of Korea (A101936). The authors thank Ji Youn Park, M.D. for image analysis assistance.

\section{Author details}

'Department of Dermatology, Ajou University School of Medicine, 164 Worldcup-ro, Yeongtong-Gu, Suwon 443-380, South Korea. ${ }^{2}$ Department of Biomedical Sciences, The Graduate School, Ajou University and Department of Microbiology, Ajou University School of Medicine, Suwon, South Korea. ${ }^{3}$ Present address: Department of Dermatology, Cheil General Hospital and Women's Healthcare Center, Dankook University College of Medicine, Cheonan, South Korea.

Received: 4 November 2014 Accepted: 20 June 2015

Published online: 02 July 2015

\section{References}

1. Direskeneli H. Autoimmunity vs autoinflammation in Behcet's disease: do we oversimplify a complex disorder? Rheumatology (Oxford). 2006;45:1461-5.

2. Pineton de Chambrun M, Wechsler B, Geri G, Cacoub P, Saadoun D. New insights into the pathogenesis of Behcet's disease. Autoimmun Rev. 2012:11:687-98.

3. Yamaguchi Y, Kurita-Ochiai T, Kobayashi R, Suzuki T, Ando T. Activation of the NLRP3 inflammasome in Porphyromonas gingivalis-accelerated atherosclerosis. Pathog Dis. 2015;73:epub. doi:10.1093/femspd/ftv011.

4. Liang L, Tan X, Zhou Q, Zhu Y, Tian Y, Yu H, et al. IL-1 beta triggered by peptidoglycan and lipopolysaccharide through TLR2/4 and ROS-NLRP3

\section{Submit your next manuscript to BioMed Central and take full advantage of:}

- Convenient online submission

- Thorough peer review

- No space constraints or color figure charges

- Immediate publication on acceptance

- Inclusion in PubMed, CAS, Scopus and Google Scholar

- Research which is freely available for redistribution

Submit your manuscript at www.biomedcentral.com/submit
C BioMed Central 